\title{
SALUD INTEGRAL DEL ADULTO MAYOR DE LA “CASA DE LOS ABUELOS ANGÉLICA ALVARADO” DEL SECTOR PANTANO ABAJO ESTADO FALCÓN
}

\author{
Sandra Romero \\ sandrar.unefm@gmail.com \\ Universidad Nacional Experimental Francisco de Miranda \\ Falcón - Venezuela \\ Jhon Jaimes \\ Johnjaimes85@gmail.com \\ Universidad Nacional Experimental Francisco de Miranda \\ Falcón - Venezuela
}

\begin{abstract}
RESUMEN
La presente investigación se planteó como propósito implementar estrategias para la Salud Integral del Adulto Mayor de la Casa de los Abuelos "Angélica Alvarado" del Sector Pantano Abajo del Municipio Miranda, Estado Falcón. La metodología utilizada fue la de Investigación Acción Participativa (IAP) aplicada a estudios sobre realidades humanas y con el empleo de técnicas como la observación participante, entrevista a informantes claves, priorización del problema con la técnica de Ranqueo, FODA y árbol del problema, lo cual permitió determinar la realidad social en la casa de los abuelos "Angélica Alvarado". Para la intervención se estableció un plan de acción para la implementación de estrategias de salud en las áreas; cardiovascular, detección de micosis superficiales y enfermedades metabólicas e infecciosas a través de la Secretaria de Salud del Estado Falcón y el Ambulatorio "Dr. Edgar Peña"; además de la realización de talleres de formación en proyectos socio-productivos por parte de la Corporación Falconiana de Turismo y de actividades físicas - recreativas por docentes del Programa de Educación Física, Recreación y Deporte de la Universidad Nacional Experimental "Francisco de Miranda" para mejorar la calidad de vida de los adultos mayores del sector Pantano Abajo. El propósito trazado de la investigación se cumplió mediante la ejecución de diferentes Fases que conllevaron al estudio y conocimiento de las problemáticas más sentidas por parte de los adultos mayores de la casa de los Abuelos "Angélica Alvarado", además se logró motivar la participación y colaboración de diferentes instituciones del Estado, la Comuna Heroína Josefa Camejo, el Consejo Comunal Territorio social I. Así como también, de la Comunidad en general del sector Pantano. La investigación permitió a través de la práctica transformadora y el cambio social resaltar la importancia de la salud integral del adulto mayor que como ciudadano activo con derechos y deberes debe ser integrado a todas las actividades de la comunidad que conlleven a mejorar su calidad de vida.
\end{abstract}

Descriptores: estrategias; participación; salud Integral; adulto mayor; calidad de vida. 


\title{
INTEGRAL HEALTH OF THE ELDERLY ADULT OF THE "HOUSE OF GRANDPARENTS ANGELICA ALVARADO" OF THE PANTANO SECTOR DOWN STATE FALCÓN
}

\begin{abstract}
The present investigation aimed to implement strategies for the Comprehensive Health of the Elderly Adult of the "Angélica Alvarado" Grandparents' House of the Pantano Sector Down of the Municipality Miranda Falcon State. The methodology used was the Participatory Action Research (IAP) applied to studies on human realities and the use of techniques such as participant observation, interviews with key informants, prioritization of the problem with the Ranqueo technique, SWOT and problem tree, Which allowed to determine the social reality in the house of the grandparents "Angelica Alvarado". For the intervention, an action plan was established for the implementation of health strategies in the areas; Cardiovascular disease, detection of superficial mycoses and metabolic and infectious diseases through the Secretary of Health of the Falcon State and the outpatient clinic Edgar Peña, in addition to the training workshops on socio-productive projects by the Falconian Tourism Corporation and Physical - recreational activities by teachers of the Physical Education, Recreation and Sports Program of the National Experimental University "Francisco de Miranda" to improve the quality of life of the elderly in Pantano Abajo. The purpose of the investigation was fulfilled through the execution of different Phases that led to the study and knowledge of the problems most felt by older adults of the house of the "Angélica Alvarado" Abuelos, also was motivated the participation and collaboration Of different institutions of the State, the Josefa Camejo Heroine Commune, the Community Council Social Territory I, as well as the Community in general of the Swamp sector. The research allowed through the transforming practice and social change to emphasize the importance of the integral health of the older adult who as an active citizen with rights and duties must be integrated to all the activities of the community that lead to improve their quality of life.
\end{abstract}

Keywords: strategies; participation; integral health; elderly: quality of life

\section{Introducción}

La sociedad muestra grandes transformaciones en diversos ámbitos, todo está inmerso en una serie de innovaciones que giran alrededor del progreso y de los avances tecnológicos. Se esta frente a un mundo globalizado donde la información se obtiene en segundos y los parámetros de vida tienden a modificarse. Venezuela no escapa a todas estas formas de cambio acelerado y se plantea 
constantemente como país nuevos retos que le permitan dar solución a los problemas y se diseñan las bases de un desarrollo acorde con las nuevas expectativas de la sociedad. En este sentido, la Universidad Nacional Experimental "Francisco de Miranda" (UNEFM), se integra al desarrollo de la región promoviendo la integración con la comunidad e incentiva situaciones que contribuyan a la articulación de los procesos necesarios que impulsen la cooperación y la solidaridad, analizada y canalizada desde escenarios de acción como lo son los Consejos Comunales. De tal manera, que en consonancia con los objetivos de la UNEFM se efectuó una intervención comunitaria en el sector Pantano Abajo para la detección de las diferentes problemáticas y en conjunto con la comunidad organizada se procedió a la ejecución de acciones que permitieron su resolución para mejorar la calidad de vida de los ciudadanos de la comunidad.

Estas acciones fueron realizadas desde el ámbito educativo e institucional entendiendo que los cambios necesitan la integración y la cooperación de diferentes entes para lograr su consecución. De esta manera, con la organización y planificación de estrategias se buscó cambiar la realidad de los adultos mayores de la casa de los abuelos "Angélica Alvarado" a través de la atención de la salud integral.

\section{FASE I: DIAGNÓSTICO}

\section{Características Generales de la Comunidad}

\section{-Aspectos Geográficos}

La Comunidad de Pantano Abajo se encuentra ubicada en el Municipio Miranda, Parroquia Santa, específicamente en la parte nor-oeste de la ciudad de Santa Ana de Coro y sus Límites son:

Norte: Calle norte y Callejón norte

Sur: Avenida Alí Primera y Calle Falcón

Este: Acera Oeste de la Avenida Miranda cruce Escuela Taller Coro

Oeste: Calle 23 de Enero 
Además la comunidad de Pantano Abajo está constituida por cinco (5) calles, de ESTE a OESTE, calle Zamora, Avenida Alí Primera, calle Falcón, calle Urdaneta y calle Miranda, y siete (7) calles de NORTE a SUR, que son: calle 23 de Enero, calle Irausquín, calle Ayacucho, calle Hospital, calle Colón, calle Federación, y Avenida Miranda. ${ }^{(1)}$

\section{-Aspectos Históricos}

Su fundación data del año 1719, cuando el Sargento Mayor Juan Damián Pérez Medina, juez subdelegado para la composición de las tierras de la ciudad de Coro, levanto el primer alindamiento de los ejidos propios y baldíos a fin de beneficiarla del real patrimonio. En base a este alindamiento de Coro, se mensuró las tierras lo cual llevo a que algunos vecinos utilizarán este sector como hatillos de ganado caprino y el sitio era conocido con el nombre de Pantano debido a las características anegadizas de sus tierras. ${ }^{(2)}$

\section{-Aspectos Culturales: Valores y tradiciones}

Es una comunidad con valores cristianos de paz, respeto y muy trabajadora, demuestran mucha empatía con los visitantes de la zona. Las fiestas patronales que se celebran son: El 11 de febrero día de la Virgen de Lourdes, 26 de Julio día de Santa Ana, además de las fiestas comunes del país como Carnaval, Semana Santa y Navidad. Es importante subrayar que en el sector Pantano Abajo quedan incluidas casi la totalidad de las edificaciones con valor arquitectónico más relevante del Casco histórico, declarado por la Organización de las Naciones Unidas para la Educación, la Ciencia y la Cultura (UNESCO), como Patrimonio de la Humanidad el día 9 de diciembre de 1993 y que además por sus valores culturales el Instituto del Patrimonio Cultural de Venezuela ha declarado a Pantano Abajo como "Bien de Interés Cultural", según resolución N003-05 de fecha 20 de febrero de 2005. ${ }^{(4)}$

\section{-Aspectos Naturales}


La comunidad de Pantano Abajo presenta las mismas características naturales de la ciudad Santa Ana de Coro, por tal motivo en el aspecto geográfico presenta un clima semiárido y con estaciones de sequía y lluvia, esta última caracterizada por pocas precipitaciones. Además la vegetación característica de esta comunidad está constituida por plantas ornamentales y vegetación xerófila. En relación a la fauna predominan los animales domésticos.

\section{-Infraestructura y Equipamiento Físico}

La configuración general de la arquitectura de las viviendas responden a un modelo unitario que corresponde a soluciones habitacionales de carácter social, con mejoras particulares. En la comunidad se encuentran viviendas que conservan la arquitectura colonial pero con daños en la infraestructura y que gran parte de las casas hechas con Adobe están enmarcadas dentro de la poligonal del Casco Histórico, formando parte del Patrimonio Histórico-Cultural del Estado Falcón. Debe señalarse que las vías de acceso al sector se encuentran asfaltadas, sin embargo presentan gran cantidad de huecos, hundimientos; además de observarse trabajos de reconexión de tuberías de aguas blancas y de aguas servidas donde no fue posteriormente restaurado el asfaltado.

Por otra parte, los servicios públicos con que cuenta esta comunidad son los siguientes:

- El Agua: Este servicio es distribuido a sus habitantes por medio de tuberías pero con días de racionamiento de acuerdo a esquema establecido por HIDROFALCÓN.

- La Electricidad: Este servicio beneficia a toda la comunidad, por cuanto disponen de postes y tendido eléctrico.

- Vías de comunicación: Las vías de acceso a la comunidad presentan irregularidades destacándose la presencia de gran cantidad de hundimientos y huecos.

- Seguridad Policial: Existe la presencia de un módulo policial quien garantiza la supervisión de actos ilícitos en el sector y sus adyacencias. 
- Transporte Público: Representado por los carritos por puesto y los buses o busetas quienes facilitan el traslado de los pasajeros de la comunidad a otros sectores del Municipio.

Pantano Abajo es una comunidad grande en extensión lo cual conlleva a que pueda presentar deficiencias en los diferentes servicios públicos.

\section{-Aspectos Sociales}

\section{Salud}

El sector de Pantano Abajo cuenta con un Barrio Adentro I, ubicado entre la calle 23 de enero y la calle $43 \mathrm{~A}$, el cual presenta daños a nivel estructural con afectación del techo y los baños, es por ello que se dificulta la atención de los pacientes que acuden a este centro de salud lo que conlleva a no ofrecer una atención de calidad a la comunidad. También existe en el sector un Ambulatorio Urbano tipo II, que tiene por nombre "Dr. Edgar Peña Acosta", el cual surge como una obra de interés social en materia de salud para beneficiar a los habitantes del sector Pantano Abajo.

\section{La Educación}

En el sector de Pantano Abajo se encuentran las misiones educativas Ribas y Sucre. Así como también, está el Complejo Educativo Pestalozzi y la Escuela Técnica Industrial Robinsoniana Coro (ETIR Coro), cuenta con simoncitos como la Escuela de Educación Inicial Josefin Leidenz, con Aldeas Universitarias ubicadas en el Complejo Pestalozzi y con Universidades Públicas como la Universidad Nacional Experimental "Simón Rodríguez", la Universidad Nacional Abierta y los Núcleos Académicos de Santa Ana y Borregales de la Universidad Nacional Experimental " Francisco de Miranda" (UNEFM), igualmente en el sector se encuentra un Instituto Educativo privado, el Colegio "Monseñor Castro". De tal manera que el sector posee institutos educativos tanto públicos como privados que permiten la inclusión de la población en el ámbito educativo en todos los niveles. 


\section{Economía}

En lo que a economía se refiere, el sector Pantano Abajo presenta un gran potencial humano el cual está representado por la población joven en su mayoría estudiantes y profesionales. Sin embargo, la falta de oportunidades de empleo en la comunidad por la no existencia de empresas o comercios incide directamente en la economía familiar lo que conlleva a que existan las siguientes características sociales:

\section{TABLA N ${ }^{\circ}$ 1. ESTRATIFICACIÓN DE LAS FAMILIAS SEGÚN CLASIFICACIÓN GRAFFAR. SECTOR PANTANO ABAJO, PARROQUIA SANTA ANA MUNICIPIO MIRANDA}

\begin{tabular}{lcc}
\hline Estrato & Frecuencia Fr & Porcentaje \% \\
\hline Estadio I Clase Alta & 0 & $0 \%$ \\
\hline Estadio II Clase Media Alta & 17 & $3.80 \%$ \\
\hline Estadio III Clase Media & 150 & $33.55 \%$ \\
\hline Estadio IV Clase Obrera & 270 & $60.40 \%$ \\
Estadio V Pobreza Extrema & 10 & $2.09 \%$ \\
\hline Total & 447 & $100 \%$
\end{tabular}

Fuente: Ambulatorio Urbano tipo II Edgar Peña 2016

Análisis: Según los datos de la tabla se obtienen los siguientes resultados: la mayor parte de la población se ubicó en el nivel socioeconómico perteneciente al estrato IV (clase obrera) con un $60.40 \%$, seguido del estrato III (Clase Media) el cual representa el $33.55 \%$, el estrato II (clase media alta) corresponde a un $3.08 \%$ y por último el estrato $\mathrm{V}$ (pobreza extrema) donde se observa un $2.09 \%$. Los resultados permiten determinar la realidad social y económica de las familias de la comunidad del Sector Pantano Abajo. 


\section{Datos Demográficos}

La comunidad del Sector Pantano Abajo agrupa aproximadamente a 2208 familias para conformar un total de 4351 personas que constituyen la población del sector, por consiguiente es importante conocer los diferentes grupos etarios que hacen vida en la comunidad lo cual permite establecer las características generales de la población.

TABLA N 2 DISTRIBUCIÓN SEGÚN EDAD Y SEXO DE LA POBLACIÓN DE PANTANO ABAJO DEL MUNICPIO MIRANDA DEL ESTADO FALCÓN AÑO 2015

\begin{tabular}{|c|c|c|c|}
\hline EDAD & MASCULINO & FEMENINO & TOTAL \\
\hline-1 & 73 & 57 & 130 \\
\hline 1 a 4 años & 96 & 71 & 167 \\
\hline 5 a 9 años & 187 & 207 & 394 \\
\hline 10 a 14 años & 279 & 281 & 560 \\
\hline 15 a 19 años & 263 & 323 & 586 \\
\hline 20 a 24 años & 208 & 194 & 402 \\
\hline 25 a 29 años & 229 & 237 & 466 \\
\hline 30 a 34 años & 176 & 187 & 363 \\
\hline 35 a 39 años & 189 & 192 & 381 \\
\hline 40 a 44 años & 87 & 74 & 161 \\
\hline 45 a 49 años & 88 & 67 & 155 \\
\hline 50 a 54 años & 73 & 68 & 141 \\
\hline 55 a 59 años & 45 & 59 & 104 \\
\hline 60 a 64 años & 92 & 141 & 233 \\
\hline 65 y mas & 49 & 59 & 108 \\
\hline TOTAL & 2134 & 2217 & 4351 \\
\hline
\end{tabular}

FUENTE: Ambulatorio Urbano tipo II “Edgar Peña”, Pantano Abajo. 2015

Análisis: Según los datos de la tabla se obtienen los siguientes resultados: La existencia de gran cantidad de población menor a 30 años lo que indica que la mayoría de los habitantes del sector Pantano Abajo son jóvenes con un total de 1454 personas, lo cual representa un $33,42 \%$ de la población total estableciéndose de esta manera de que existe una gran cantidad de población activa en el sector de la comunidad además de estar conformada por una población expansiva con alta natalidad, esto se debe en primer lugar a que la mayor parte de la población se encuentra comprendida entre los 0 y 15 años de edad. También en la comunidad hay 445 adultos mayores que necesitan de la atención y participación activa en la comunidad como garantes de la tradición y costumbres del sector. Sin embargo, se destaca que se está realizando en la actualidad una nueva data de la población de la comunidad la cual es efectuada por las trabajadoras sociales del Ambulatorio "Dr. Edgar Peña". 


\section{Aspectos Políticos: Comuna Heroína Josefa Camejo}

El sector de Pantano Abajo se encuentra organizado en siete Consejos Comunales: Gran Mariscal de Ayacucho, Territorio Social I, Pantano Abajo III, Pantano Abajo IV, Parcelamiento Josefa Camejo, Corazón Valiente (sector III) y Santa Ana sector I. Ahora bien, estos Consejos Comunales están adscritos a la Comuna "Heroína Josefa Camejo", la cual se encuentra ubicada en la ciudad de Santa Ana de Coro, Parroquia Santa Ana del Municipio Miranda y su ámbito geográfico comprende los sectores Pantano Abajo (I, II, III y IV), Parcelamiento Josefa Camejo y Parcelamiento Cástulo Mármol Ferrer. Además sus linderos son; por el norte con el aeropuerto "José Leonardo Chirinos" y Variante Sur, por el sur con calle Falcón y Avenida Alí Primera, por el este Avenida Francisco de Miranda y por el oeste Variante Norte. La Comuna agrupa a 2208 Familias para conformar un total de 4351 personas que constituyen la población de la comuna. Esta comuna persigue como meta alcanzar la solución de diversos problemas que se presentan en la comunidad y que están relacionados con educación, salud, deporte, alimentación, seguridad, embaulamiento de corrientes de aguas pluviales y quebradas, déficit de viviendas, bajos niveles de producción, deficiencia en la recolección de desechos sólidos, carencia de sistemas de financiamiento, falta de asesorías para créditos en diferentes rubros, ausencia de ayudas a adultos mayores y deficiencia de medicamentos en los ambulatorios. ${ }^{(5)}$

\section{- PERCEPCIÓN INICIAL}

En el proceso de investigación se destaca la necesidad de realizar diversas acciones que conduzcan a obtener información pertinente. Existen recursos que permiten realizar el diagnóstico efectivo del contexto en estudio y cuyos resultados dan una visión de la problemática existente y de lo que se quiere cambiar de la realidad. Por consiguiente; "El investigador habrá de seleccionar un campo concreto, porque la investigación científica no se realiza en términos generales sino definiendo problemas específicos dentro de áreas particulares del conocimiento." ${ }^{(6)}$ Por consiguiente, en el abordaje de la comunidad del Sector 
Pantano Abajo se procedió a contactar a los miembros de la Comuna "Heroína Josefa Camejo", realizar una observación participante y se entrevistó a informantes claves en la comunidad que permitieran hacer un diagnóstico participativo lo cual conllevo a la identificación de las problemáticas.

\section{- Identificación de los Problemas}

El primer contacto con la comunidad se realizó con el Sr. Diomar Medina, quién es representante parlamentario de la Comuna "Heroína Josefa Camejo" y en este encuentro se planteó realizar una intervención en la comunidad que tenía como propósito recabar información para intentar aplicar medidas de solución a problemas o necesidades que se presentan en la realidad social. En este sentido se entiende que; "La intervención es la introducción de un agente externo para modificar el funcionamiento de un proceso o sistema; desde el punto de vista social, la intervención se aplica a contextos sociales en los que se requiera la modificación de alguna situación determinada" ${ }^{(6)}$. Posteriormente se realizó recorridos por la comunidad de Pantano Abajo en busca de informantes claves de manera de conocer a través de entrevistas no estructuradas las diversas problemáticas que se presentan en los sectores que corresponden geográficamente a la comuna, encontrando en el Ambulatorio Urbano tipo II "Edgar Peña" gran cantidad de personas del sector por ser el centro de salud más concurrido lo cual llevo a obtener información pertinente sobre la comunidad. Entre los informantes claves esta la Licenciada Hilda Sánchez quien es la trabajadora Social del ambulatorio, quien fue muy receptiva suministrando información sobre la historia de la comunidad, distribución de la población según sexo y edad, además de los estratos sociales de la población en estudio. De igual manera, se conversó con la Licenciada Xiomara Navarro jefe de enfermeras del ambulatorio, quién suministro información sobre las enfermedades presentes en el sector y las actividades del Ambulatorio en relación a la Atención Primaria de Salud. También se entrevistó a la Sra. Gladys Sambrano quien reside en el sector quien aportó información relevante sobre la comunidad, especialmente haciendo 
referencia a problemáticas sobre inseguridad, asfaltado de las calles y la deficiencia de medicamentos en el Ambulatorio entre otras.

Consecuentemente, se planificó un segundo encuentro con la comunidad en el que se llevó a cabo la asamblea de ciudadanos y ciudadanas de la Comuna en estudio, donde se produjo una reunión extraordinaria por parte del equipo de profesores de la UNEFM y la comunidad. Se realizó la presentación de los profesores y se planteó el propósito de la asamblea, el cual era conocer las dificultades o problemas que presenta la comunidad en general. Por consiguiente, los miembros participantes del Consejo Comunal y de la comunidad exponen diferentes problemáticas, entre las que están: Déficit de viviendas, calles deterioradas por falta de asfaltado, deficiencia en el servicio de recolección de basura ( desechos sólidos), ausencia de ayuda a los adultos mayores, falta de financiamiento para mejoras en las viviendas.

Luego de la exposición de las situaciones por parte de los miembros de los consejos comunales y de la comunidad se obtuvo 4 situaciones problemáticas y se procedió a la Técnica de Ranqueo de Problemas, se les explicó a los asistentes la metodología a implementar, la cual consistió en hacer preguntas específicas para cada una de las variables correlacionándolas con el problema.

TABLA N 3 MATRIZ DE PRIORIZACIÓN DE PROBLEMAS

\begin{tabular}{|c|c|c|c|c|c|c|c|c|}
\hline Problemas & A & B & C & D & $E$ & $\mathbf{F}$ & Total & № \\
\hline $\begin{array}{l}\text { Poca Celeridad del proyecto de viviendas por parte } \\
\text { de la comuna }\end{array}$ & 2 & 2 & 1 & 1 & 1 & 1 & 8 & 3 \\
\hline $\begin{array}{l}\text { Ausencia de ayuda a los adultos mayores a nivel de } \\
\text { salud, deportivo y económico }\end{array}$ & 2 & 2 & 2 & 1 & 2 & 2 & 11 & 1 \\
\hline Deterioro de algunas calles por Falta de asfaltado & 1 & 2 & 1 & 1 & 1 & 1 & 7 & 4 \\
\hline $\begin{array}{l}\text { Deficiencia en el servicio de Recolección de basura } \\
\text { ( desechos sólidos) }\end{array}$ & 2 & 2 & 1 & 2 & 2 & 1 & 10 & 2 \\
\hline
\end{tabular}

Leyenda:

A: Frecuencia: Más - Menos - Repetir

B: Tendencia: Ascendente - Descendente

C: Gravedad: Mayor o Menor

D: Disposición de recursos: Con Factibilidad de solución de acuerdo a los recursos existentes

E: Vulnerabilidad: Más o Menos

F: Coherencia: Coherente con la misión de planificadores 
De 0 a 2 Puntos:

2: Cuando el criterio se cumple a cabalidad

1: Cuando el criterio se cumple en parte

0 : Cuando el Criterio no se cumple

En consecuencia se obtiene como resultado que el problema priorizado fue la falta de ayuda a los adultos mayores específicamente de la Casa de los Abuelos "Angélica Alvarado", que se encuentra ubicada en el sector Pantano Abajo y que geográficamente pertenece al Consejo Comunal Territorio Social I y en la cual se realizan las actividades gerontológicas de los adultos mayores de la comunidad. De este modo, una vez conocido el Consejo Comunal para la intervención se realizó un diagnóstico participativo comunitario directamente con los miembros de la casa del adulto mayor, quienes son el centro de intervención social para esta investigación. Visto de esta forma el diagnóstico participativo comunitario es un proceso permanente que conlleva a la reflexión y por medio del cual se intenta conocer de manera general la situación y los aspectos más relevantes de una comunidad específica ${ }^{(6)}$. Es por ello que, este proceso se llevó a cabo a través de la recolección, procesamiento de datos y análisis de la información obtenida, con el propósito de definir las prioridades, además de tener en cuenta la factibilidad del estudio, planificación, acciones y estrategias a implementar.

\section{CASA DE LOS ABUELOS "ANGÉLICA ALVARADO"}

En esta perspectiva, se tiene que la Casa de los Abuelos "Angélica Alvarado", está integrada por un club de Adultos Mayores que lleva por nombre "Mis Años Dorados". Se encuentra ubicada en el Sector Pantano Abajo, específicamente en la Calle Colón entre Calle Miranda y Urdaneta del Municipio Miranda, Estado Falcón. Debe señalarse que el circulo de la tercera edad "Mis años dorados", inicio sus actividades el 22 de Octubre de 1997; en el Ambulatorio "Edgar Peña Acosta" del sector Pantano Abajo formado por el servicio de Promoción Social del Ambulatorio y el Departamento de Gerontología de la Alcaldía del Municipio Miranda. Desde sus inicios contó con una directiva que estaba presidida por el señor Luis Figueredo (Q.E.P.D), luego siguió el señor 
Carlos Romero (Q.E.P.D), después José Ramírez, luego Enrique García y Elva de Pachano, actualmente en la presidencia se encuentra el Sr. Juvenal Medina. Se destaca que al cumplir 10 años el club estreno sede propia y lleva por nombre Casa de los Abuelos "Angélica Alvarado", nombre que hace honor a una señora que a su corto paso por el circulo dejo profunda huella en los que la conocieron. La casa que da cobijo al círculo de la tercera edad del sector Pantano Abajo, perteneció a la familia Sierra Ramírez, vecinos del sector, la cual fue adquirida por ASOAVI, para ser utilizada única y exclusivamente por el círculo de la tercera edad. La casa de los abuelos "Angélica Alvarado" tiene entre sus objetivos:

- Potenciar la promoción de envejecimiento sano y activo como proceso de optimización de las oportunidades de salud, participación y la seguridad; con el fin de mejorar la calidad de vida a medida que las personas envejecen.

- Optimizar la prevención y protección social de personas mayores con necesidades.

- Promover la gerontología positiva dedicada al énfasis de los aspectos positivos que la vejes y el envejecimiento lleva consigo. ${ }^{(7)}$

Cabe considerar por otra parte, que los 60 adultos mayores que hacen vida en la Casa de los abuelos "Angélica Alvarado", de acuerdo a información suministrada por los habitantes del sector (informantes claves) y los miembros de los diferentes consejos comunales, necesitan de atención para la solución de las problemáticas que allí se presentan y que no permiten cumplir con sus objetivos, por lo que se requería la atención y el abordaje a nivel de ciertos ámbitos o aspectos que permitieran mejorar su calidad de vida.

En consecuencia, se procedió a la construcción de la Matriz FODA del problema priorizado como número uno a través de la técnica de Ranqueo. En este sentido, se realizaron una serie de preguntas oportunas y pertinentes, a partir de las cuales se elaboraron las estrategias de intervención para posteriormente lograr el 
plan de acción y la búsqueda de la solución al problema. Siendo las interrogantes formuladas las siguientes:

- ¿Cuál es la situación actual del problema?

- ¿Qué se ha hecho hasta ahora sobre este problema?

- ¿Quiénes han intervenido en el problema?

- ¿Con que fortalezas y oportunidades se cuenta para la solución de este problema?

- ¿Cuáles son las debilidades y amenazas ante este problema?

\section{MATRIZ FODA}

Las fortalezas son: El consejo Comunal Territorio Social I, los miembros voluntarios de la comunidad, el potencial por parte de los adultos mayores en actividades económicas relacionadas con el arte culinario, lencería y dulcería. La Casa de los Abuelos cuenta con una infraestructura amplia, cómoda, dotada de equipos electrónicos y tecnológicos para el esparcimiento y bienestar de todos los adultos mayores que hacen vida en ella.

Las oportunidades son: La intervención de los profesores de la UNEFM y el apoyo de la Licenciada en gerontología.

> Las debilidades son: No hay vinculación con el Ambulatorio "Edgar Peña" como grupo social para la Atención Primaria de Salud que permita el diagnóstico y el control de las patologías crónicas e infecciosas de los adultos mayores, la falta de asesoría para la elaboración de proyectos socio productivos, no realizan actividad física y de recreación además de la presencia de daños a la infraestructura de la casa del adulto mayor.

Las amenazas que presentan son: El factor tiempo, la apatía de un grupo de la comunidad, la falta de integración de la coordinación interinstitucional en el área de la salud para el abordaje de la salud integral de los miembros de la casa del adulto mayor "Angélica Alvarado" y de la inseguridad en el sector. Sin embargo, a pesar de las debilidades y 
amenazas que presentan se toman en cuenta aquellas teniendo en consideración el factor tiempo y la factibilidad. Posteriormente se realizó Mesas Socializadoras con los miembros de la Casa de los Abuelos, esto con la finalidad de conocer las causas que generan los problemas ya detectados en el diagnóstico participativo y sus posibles consecuencias, esto se logró gracias a la técnica del Árbol del Problema.

\section{GRAFICO №1 ARBOL DEL PROBLEMA}

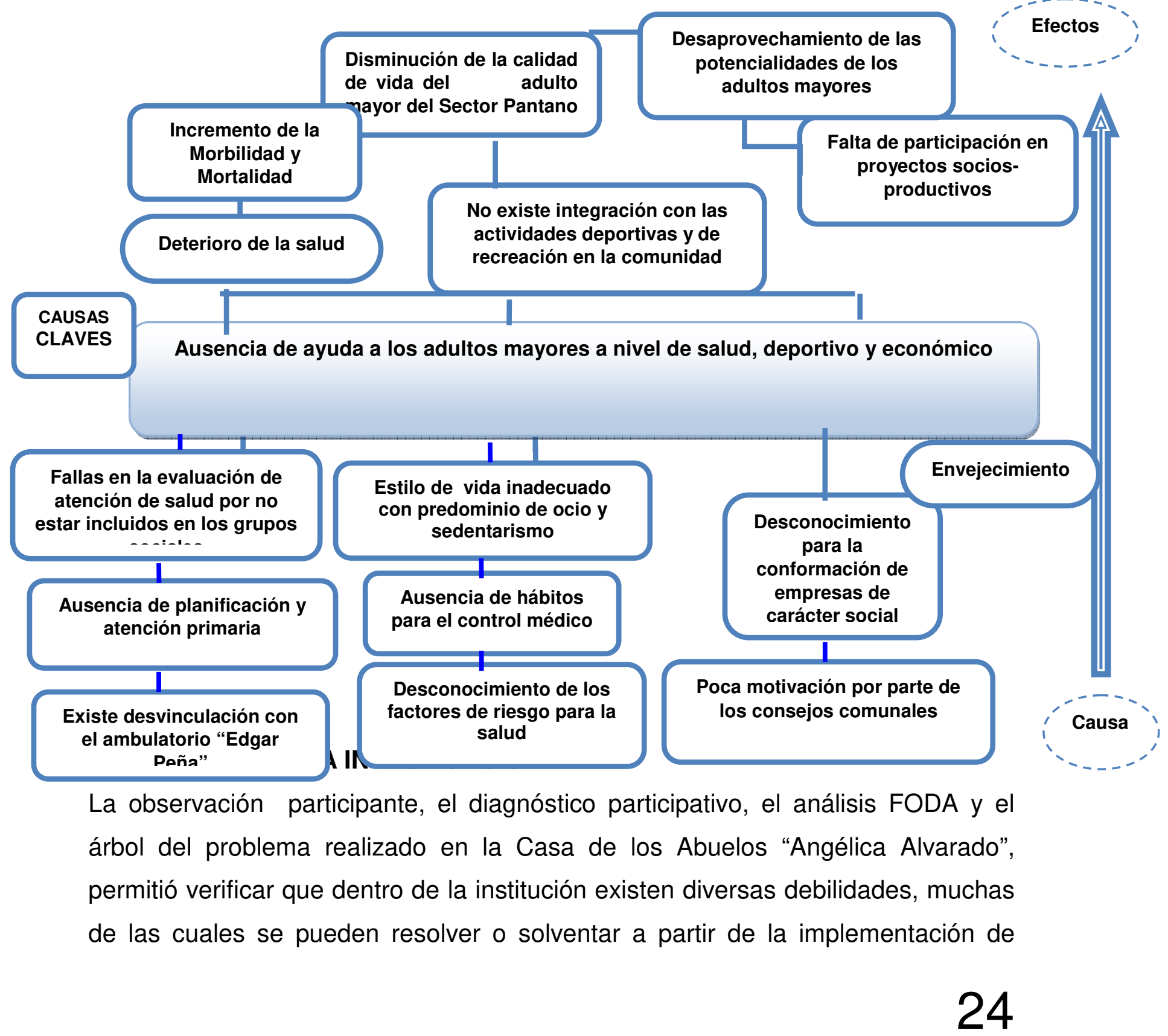


estrategias y planes específicos, es decir, se pudo palpar de manera directa las diferentes situaciones que afectaban el cumplimiento de los objetivos de la Casa de los Abuelos y se buscó desarrollar experiencias que permitieran implementar cambios significativos para transformar la realidad social a través del trabajo en conjunto con la Comuna "Heroína Josefa Camejo", el Consejo Comunal Territorio social I y la Directiva de la casa de los abuelos Angélica Alvarado". Es por ello, que se planteó como propuesta cambiar la realidad existente dentro de la Casa de los Abuelos “Angélica Alvarado", en cuanto a la ausencia de ayuda a los adultos mayores en aspectos relacionados con la falta de estrategias de salud que permitan la atención primaria de salud, la realización de actividades físicas y la formación en proyectos socio-productivos. Por consiguiente, se trató de cambiar esta situación a través acciones concretas entendiendo que a través de un ambiente psicosocial adecuado y contando con la Atención Primaria de Salud, los recursos y la formación pertinente, los adultos mayores pueden mejorar su calidad de vida, se buscó transformar la realidad.

\section{PROPÓSITOS DE LA INVESTIGACIÓN}

\section{> PROPÓSITO GENERAL}

Implementar estrategias para la salud integral del adulto mayor de la casa de los abuelos "Angélica Alvarado" del sector Pantano Abajo Estado Falcón.

\section{$>$ PROPOSITOS ESPECIFICOS}

-Efectuar un diagnóstico en la casa de los abuelos "Angélica Alvarado" que conlleve a detectar las problemáticas más relevantes.

- Aplicar estrategias de salud en las áreas de Microbiología, Cardiovascular, Endocrino y Enfermedades metabólicas en la casa de los abuelos "Angélica Alvarado".

- Realizar actividades físicas y de recreación para los adultos mayores de la casa de los abuelos "Angélica Alvarado" para fomentar hábitos de vida saludable. 
-Efectuar talleres de formación en Proyectos Socio-Productivos para los adultos mayores de la casa de los Abuelos "Angélica Alvarado".

\section{FASE II: CONTEXTO TEÓRICO}

\section{EL ENVEJECIMIENTO}

Se puede definir el envejecimiento como la pérdida de la capacidad del organismo a adaptarse al medio ambiente, lo que requiere especial atención desde el punto de vista de la salud. De igual manera, esta etapa comprende un amplio conjunto de procesos biológicos, psicológicos y sociales relacionados con la vida después de la edad madura, incluyendo aspectos positivos y negativos; no se limita al declive de las estructuras y funciones corporales. Las secuelas en el funcionamiento y la participación social, también involucra aspectos como procesos patológicos previos, ganancias psicológicas, es decir, experiencias y ganancias sociales relacionadas con el envejecimiento activo en las etapas avanzadas de la vida ${ }^{(8)}$. Según Ribera( 2009), es imposible establecer un corte de edad a partir del cual una persona deba ser considerada adulto mayor, aunque este solo es admisible en dos situaciones: Una, por efectos administrativos como la jubilación, y la otra, epidemiológica, es decir, para establecer puntos de corte para estudios de prevalencia o de intervención en determinado proceso a estudiar, pues como se ve, ambas situaciones son arbitrarias y orientadas por más que puedan ser necesarias por los objetivos que persiguen. El envejecimiento tiene que analizarse de forma dinámica por ser un proceso que se inicia con el nacimiento pero que a partir de los 30 años hay un momento donde se alcanza la plenitud, y existe un cambio donde los procesos catabólicos superan a los procesos anabólicos, entonces hay una pérdida de los mecanismos de reserva del organismo, lo que determina un aumento de la vulnerabilidad ante cualquier tipo de agresión, lo cual implica por lo tanto mayores probabilidades de padecer enfermedades y morir. ${ }^{(8)}$ 


\section{LA SALUD EN EL ADULTO MAYOR}

Los problemas y limitaciones que afectan al adulto mayor son originados por las diversas patologías que los afecta, los cambios fisiológicos que se van presentando como consecuencia del proceso natural del envejecimiento donde juega un papel muy importante la condición general, tanto física como mental, a esta edad. ${ }^{(10)}$ En consecuencia, según Rose (1991), numerosos son los cambios fisiológicos que van presentándose durante el proceso de envejecimiento. Desde el punto de vista general se produce un aumento de la grasa corporal de un $14 \%$ a un $30 \%$ y una disminución del agua corporal total, sobre todo en el compartimento intracelular, por lo que hay mayor tendencia a la deshidratación, en algunos casos al sobrepeso y en una mayor permanencia de las drogas lipofílicas. Por otra parte la disminución de la masa y de la fuerza muscular no le permite enfrentar con éxito los requerimientos de mayor fortaleza y de mayor rapidez en la movilidad. La disminución de la densidad ósea hace más frágiles sus huesos (osteopenia/osteoporosis) por lo que pueden fracturarse más rápidamente, produciéndose limitación funcional y caídas. ${ }^{(11)} \mathrm{De}$ igual manera a nivel cardiovascular, se produce una disminución de la "compliance" vascular arterial, con la subsecuente elevación de la presión arterial (sin llegar a grados de hipertensión) y disminución del flujo sanguíneo con el resultado de isquemia de algunos órganos (corazón, cerebro, riñones). Igualmente, se produce una disminución de la respuesta b-adrenérgica con menor capacidad del bombeo cardiaco y de cronotropismo. Las consecuencias clínicas son la disminución del gasto cardiaco, la disminución de la frecuencia cardiaca máxima, elevación de la presión arterial sistólica, aumento de la presión de pulso, vulnerabilidad a la Hipotensión Arterial, pobre respuesta compensatoria a cambios de la presión arterial, susceptibilidad al síncope e hipo-perfusión orgánica. ${ }^{(12)}$

Por otra parte en el aparato respiratorio del adulto mayor hay una disminución de la elasticidad pulmonar y un aumento de la rigidez torácica, que no permiten una buena contracción-distensión pulmonar, con el consecuente déficit en el intercambio de gases. ${ }^{(13)}$ También el envejecimiento involucra cambios metabólicos 
que pueden inducir a una Diabetes Mellitus 2 (DM2). ${ }^{(14)}$ En este sentido hay que entender que el adulto mayor presenta un incremento en la posibilidad de desarrollar DM2 debido al declive natural de las células beta del páncreas, a la insulino-resistencia, a la menor actividad física, a la alimentación inadecuada y a la presencia de otras enfermedades que empeoran el pronóstico y hacen complejo el tratamiento. Es por ello que en este grupo etario, se hallan las complicaciones propias debido a la merma de la funcionalidad orgánica, así como en muchos casos, la cognitiva, sumado a las complicaciones crónicas de la enfermedad de base. El adulto mayor con diabetes presenta características especiales debido a su fragilidad y a la dificultad de adaptación a los cambios, que lo hacen susceptible de complicaciones y errores a la hora de realizar el tratamiento propuesto. Por tal razón, el equipo de salud que lo acompaña debe reforzar en forma constante, paciente y paulatina los diversos elementos de la educación a todo el entorno familiar de manera de lograr un aceptable control metabólico y de las enfermedades que lo acompañan y obtener la mejor calidad de vida posible. ${ }^{(14)}$

\section{MICOSIS SUPERFICIALES}

Dentro del proceso de envejecimiento del adulto mayor existe por ende un envejecimiento cutáneo que se da tanto a nivel de la epidermis, como de la dermis y del tejido subcutáneo, el cual disminuye su contenido de grasa y de los apéndices cutáneos. ${ }^{(15)} \mathrm{A}$ nivel de la epidermis se produce una disminución de los melanocitos, de las células de Langerhans, de la membrana basal, de los keratinocitos, que trae como consecuencia la disminución de la cicatrización, de la foto-protección, de la producción de vitamina $D$, de las reacciones de hipersensibilidad, de la adhesión epidermis-dermis y un incremento en la generación de ampollas. Así mismo, a nivel de la dermis se produce una disminución de colágeno y de la elastina, igualmente la reducción de la grasa subcutánea trae como consecuencia la disminución de la protección mecánica. ${ }^{(15)}$ Es importante destacar que en la edad avanzada todas las capas de la piel se encuentran alteradas además, el estrato córneo es más delgado, sobre 
todo en zonas expuestas, tiene menor contenido en agua y mayor velocidad de descamación y la epidermis es más delgada. Cabe considerar, que las lesiones cutáneas más frecuentes en los adultos mayores son la dermatitis eccematosa, las micosis, el prurito, las infecciones bacterianas, las infecciones virales, y como en otros procesos infecciosos, se produce una respuesta febril e inflamatoria más pobre, que puede enmascararse en el cuadro clínico general. Por lo que es más frecuente su diagnóstico en estadios avanzados, la evolución es más tórpida y las complicaciones, como la aparición de bacteriemia y sepsis, son más frecuentes. ${ }^{(16)}$ En este sentido cabe poner en relieve, que las dermatofitosis o tiñas son cada vez más frecuentes en ancianos. Se presentan como placas eritematoes camosas que pueden presentarse en diferentes partes del cuerpo, inglés, pies (distintos patrones: hiperqueratosis, eccematosos o intertriginosa muy pruriginosa) y uñas. Además la onicomicosis es más frecuentes en las personas de edad avanzada y en varones, y puede ser la puerta de entrada de otras infecciones de la piel, sobre todo en personas diabéticas, como úlceras y erisipela. ${ }^{(16)}$

\section{ENVEJECIMIENTO EXITOSO}

Dentro del envejecimiento se encuentra el término envejecimiento exitoso, que se viene utilizando en los últimos años en todos los congresos como tema de conferencia. En este sentido se destaca al médico norteamericano, el Dr. William Hall, presidente del American College of Physician, quien hace referencia al tema en sus conferencias y plantea que el tener una vejez exitosa depende exclusivamente del individuo, lo cual se relaciona con aspectos; como se alimenta, si mantiene actividad física regular y continua, evitando el sedentarismo, si mantiene antes de los 75 años el peso dentro de valores ideales, si controla las enfermedades crónicas, como la diabetes y la hipertensión. Por consiguiente, cómo será el tipo de envejecimiento o cómo envejeció el organismo del individuo depende de muchas variables y factores, como el estilo de vida, las costumbres culturales, la alimentación, factores psicológicos y sociales del entorno de la persona. Es por ello que, el envejecimiento activo "es el proceso de optimización 
de las oportunidades de salud, participación y seguridad, con el objetivo de mejorar la calidad de vida en el transcurso del envejecimiento". ${ }^{(18)}$ Esta visión propone el envejecimiento como una experiencia positiva tanto para el individuo como para la comunidad, e incluye la participación de la sociedad de acuerdo con sus necesidades, deseos y capacidad. Por lo tanto no se debe pasar por alto que activo se refiere justamente a la participación en las gestiones sociales, económicas, culturales, espirituales y civiles del envejecimiento. Conviene destacar que mantener la autonomía e independencia de los adultos mayores de una comunidad durante el proceso de envejecimiento debe ser una meta fundamental para las naciones, pero también debe envolver activamente a familiares, amigos, colegas de trabajo y vecinos, pues la carga social y económica cuando estos se tornan dependientes es muy grande. ${ }^{(18)}$ Por tal motivo, el envejecimiento activo es un paso adelante del envejecimiento saludable, donde se reconocen los derechos humanos de los adultos mayores con los principios de independencia, participación, dignidad, asistencia y autorrealización, tornándose en una obligación la implementación de estrategias que permitan la promoción de planes de salud en las comunidades para valorar la participación del adulto mayor como ciudadano.

\section{TEORÍA INVESTIGACIÓN - ACCIÓN}

Kurt Lewis fue el primero en utilizar el término investigación acción en el año de 1951, a través del cual se describe una forma de investigación que permite combinar el enfoque experimental de las ciencias sociales con programas que involucren acciones de tipo social y de esta manera se pueda responder y dar solución a problemas sociales. En este sentido, cabe poner en relieve que la teoría de la acción establece la importancia de las actividades dentro del proceso de investigación. Plantea que el conocimiento práctico no se constituye en el objetivo único de la investigación acción sino que por el contrario representa el comienzo debido a que lo que se busca es la concientización de una problemática que se instituye como idea central y meta de la investigación, no solo para la producción 
de conocimientos sino además como experiencia concreta de acción. ${ }^{(19)} \mathrm{La}$ Investigación Acción-Participativa promueve experiencias sociales que favorecen la solución de problemáticas existentes en las comunidades asociado a la generación de conocimientos, esto se realiza a partir de la aplicación de categorías científicas para el mejoramiento y comprensión de la realidad, contribuyendo de esta forma a generar cambios sociales que permitan mejorar las condiciones a través de la integración de la universidad con las comunidades y de esta manera favorecer así los procesos que contribuyan a mejorar la calidad de vida de los ciudadanos con la implementación de metodologías de investigación que conlleven al cambio de la realidad pero con la participación de los miembros de la propia comunidad. ${ }^{(20)}$

\section{CONTEXTO METODOLÓGICO}

\section{PARADIGMA DE LA INVESTIGACIÓN}

El paradigma científico que guío la presente investigación: Salud integral del adulto mayor de la casa de los abuelos "Angélica Alvarado" del sector Pantano Abajo Estado Falcón, es el Paradigma Critico Reflexivo, ", través del cual se logran pensamientos realistas y un punto de vista subjetivo. Este parte del hecho de que la vida social es dialéctica, lo cual conlleva a que su estudio debe abordarse desde la dinámica del cambio social. ${ }^{(20)} \mathrm{El}$ paradigma crítico promueve la crítica reflexiva en los heterogéneos procesos que involucra el conocimiento como construcción social de igual forma, este paradigma también induce a la crítica teniendo en cuenta la transformación de la realidad pero basándose en la práctica y el sentido. ${ }^{(20)}$ Koetting, (1984), expone lo siguiente: "Con el paradigma crítico -reflexivo se busca que la práctica se modifica teórica y prácticamente, y el proceso de toma de decisiones está centrado en la capacidad crítica del investigador". 


\section{FASE IV. TÉCNICAS DE RECOLECCIÓN DE DATOS}

Dentro del proceso de investigación es primordial tener presente las diferentes técnicas e instrumentos a utilizar para la recolección de datos o de información que permitan guiar y facilitar el trabajo, y contribuyan con el logro de los objetivos planteados. Bautista (2004), expone que los instrumentos de recolección de datos: "Son los medios que permiten observar y registrar característica, conductas, entre otras.; y en general que se desea obtener en una situación educativa a investigar, evaluar o supervisar". En la presente investigación se programó como técnicas de recolección de datos: La observación participante, la entrevista no estructurada a partir de preguntas abiertas, técnica de Ranqueo, Matriz Foda, Árbol del Problema y como instrumento los registros descriptivos.

Seguidamente se utilizó la observación participante que es una técnica que permite apreciar la realidad tal como se presenta, además de permitir al investigador establecer relaciones abiertas con los informantes e interactuar con los sujetos de estudio.

En la investigación se utilizó la observación participante a través de la cual se obtuvo gran información y permitió precisar las diferentes problemáticas presentes en el Sector Pantano Abajo, que corresponde geográficamente al plan de acción de la Comuna "Heroína Josefa Camejo". De igual manera, se utilizó como técnica de recolección de datos la entrevista, la cual es una técnica que se fundamenta en el diálogo que se establece entre dos o más personas, que permite obtener informaciones relevantes de las fuentes primarias, en este caso de informantes claves como los parlamentarios de la comuna "Heroína Josefa Camejo", los habitantes del sector Pantano Abajo, el personal administrativo, medico, enfermeras, obreros y pacientes del ambulatorio urbano tipo II "Edgar Peña" ubicado en el sector Pantano Abajo. Asimismo, se utilizó la entrevista como elemento de recolección de información aplicada a los diversos miembros de la comunidad y la casa de los abuelos "Angélica Alvarado" lo cual permitió la realización de un análisis FODA y el Árbol de Problemas, de manera de conocer las inquietudes y problemáticas además de permitir la integración con el grupo 
investigado y poder conformar grupos de trabajo para dar solución a la problemática planteada. Se utilizaron los registros descriptivos como instrumento que permiten registrar en forma fidedigna y eficaz los diversos acontecimientos que tienen lugar durante el proceso de investigación y garantizan la información obtenida. A través de los registros descriptivos se detallan las diferentes situaciones y acciones llevadas a cabo durante el proceso de investigación en el sector Pantano Abajo, específicamente en la casa de los Abuelos "Angélica Alvarado". Asimismo se utilizó las fotografías como medios para evidenciar cada estrategia efectuada durante la intervención en la casa de los abuelos "Angélica Alvarado".

\section{FASE V.PLAN DE ACCION}

El presente trabajo de investigación implicó la realización y coordinación de una serie de estrategias y actividades que conllevaron a alcanzar los propósitos planteados a través de la ejecución del Plan de Acción: 
SALUD Y VIDA. Revista Arbitrada Interdisciplinaria de Ciencias de la Salud

. Año I. Volumen 1. Enero - Junio 2017. Santa Ana de Coro, Venezuela.

Hecho el depósito de Ley: FA2016000010. ISSN: 2610-8038

FUNDACIÓN KOINONIA (F.K). Santa Ana de Coro, Venezuela.

\section{CRONOGRAMA DE APLICACION DE ESTRATEGIAS}

\begin{tabular}{|c|c|c|c|c|c|c|}
\hline \multirow{2}{*}{$\begin{array}{c}\text { PROPÓSIT } \\
\text { O }\end{array}$} & \multirow{2}{*}{ ESTRATEGIA } & \multirow{2}{*}{ ACTIVIDAD } & \multicolumn{2}{|c|}{ RECURSOS } & \multirow{2}{*}{$\begin{array}{l}\text { LUGAR DE } \\
\text { EJECUCIÓN }\end{array}$} & \multirow{2}{*}{ RESPONSABLES } \\
\hline & & & MATERIALES & HUMANOS & & \\
\hline \multirow[t]{3}{*}{$\begin{array}{c}\text { Efectuar } \\
\text { diagnóstico } \\
\text { en la Casa } \\
\text { de los } \\
\text { Abuelos } \\
\text { "Angélica } \\
\text { Alvarado" } \\
\text { que } \\
\text { conlleve a } \\
\text { detectar las } \\
\text { problemátic } \\
\text { as más } \\
\text { relevantes. }\end{array}$} & $\begin{array}{l}\text {-Contactar a los miembros } \\
\text { de la Comuna Heroína } \\
\text { Josefa Camejo del sector } \\
\text { Pantano Abajo. }\end{array}$ & $\begin{array}{l}\text { Encuentro con el Sr. Diomar Medina } \\
\text { Parlamentario de la Comuna Heroína } \\
\text { Josefa Camejo del Sector Pantano } \\
\text { Abajo para informar sobre la } \\
\text { intención de realizar en el sector una } \\
\text { intervención comunitaria como } \\
\text { actividad de extensión dentro del } \\
\text { programa de formación académica de } \\
\text { la UNEFM y comenzar con la } \\
\text { observación participante en la } \\
\text { comunidad. }\end{array}$ & $\begin{array}{l}\text { Cuaderno de Notas. } \\
\text { Lápiz. } \\
\text { Cámara Fotográfica. }\end{array}$ & $\begin{array}{l}\text { Investigadores y miembros } \\
\text { de la Comuna heroína } \\
\text { Josefa Camejo. }\end{array}$ & $\begin{array}{l}\text { Sector Pantano Abajo. } \\
\text { Comuna Heroína } \\
\text { Josefa Camejo }\end{array}$ & $\begin{array}{l}\text { Docentes de la } \\
\text { UNEFM y Miembros } \\
\text { de la Comuna Heroína } \\
\text { Josefa Camejo. }\end{array}$ \\
\hline & $\begin{array}{l}- \text { - Visitar a la } \\
\text { comunidad de Pantano } \\
\text { Abajo para realizar } \\
\text { observación participante y } \\
\text { entrevista a informantes } \\
\text { claves. } \\
\text { Asamblea de convocar unadanos y } \\
\text { ciudadanas para conocer } \\
\text { problemáticas.- }\end{array}$ & $\begin{array}{l}\text {-Se realizó recorrido por la la } \\
\text { comunidad y se entrevistó a } \\
\text { informantes claves. } \\
\text { - Se efectuó asamblea de ciudadanos } \\
\text { y ciudadanas con el fin de conocer } \\
\text { las problemáticas, necesidades y y } \\
\text { potencialidades de la comunidad a } \\
\text { través de la técnica de Ranqueo de } \\
\text { problemas. }\end{array}$ & $\begin{array}{l}\text { Cuaderno de Notas. } \\
\text { Lápiz. } \\
\text { Cámara Fotográfica. }\end{array}$ & $\begin{array}{l}\text { Investigadores, miembros } \\
\text { de la comunidad del sector } \\
\text { Pantano Abajo y los } \\
\text { representantes de la } \\
\text { Comuna Heroína Josefa } \\
\text { Camejo. }\end{array}$ & Sector Pantano Abajo & $\begin{array}{l}\text { Docentes de la } \\
\text { UNEFM y miembros } \\
\text { de l C Comuna Heroína } \\
\text { Josefa Camejo. }\end{array}$ \\
\hline & 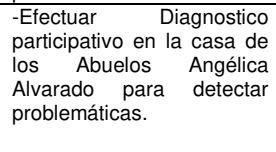 & $\begin{array}{l}\text { - Se realizó diagnostico social } \\
\text { participativo en la Casa de los } \\
\text { abuelos Angélica Alvarado para } \\
\text { conocer las problemáticas a través de } \\
\text { la Matriz de Jerarquización de } \\
\text { Problemas. }\end{array}$ & $\begin{array}{l}\text { Cuaderno de notas. } \\
\text { Lápiz. } \\
\text { Hojas blancas. } \\
\text { Cámara Fotográfica. }\end{array}$ & $\begin{array}{l}\text { Investigador, miembros de } \\
\text { la casa de los abuelos } \\
\text { Angélica Alvarado y los } \\
\text { miembros del Consejo } \\
\text { Comunal Territorio social I. }\end{array}$ & $\begin{array}{l}\text { Casa de los Abuelos } \\
\text { Angélica Alvarado, } \\
\text { callen Colón, sector } \\
\text { Pantano. }\end{array}$ & $\begin{array}{l}\text { Docente de la UNEFM } \\
\text { y miembros del } \\
\text { Consejo Comunal } \\
\text { Territorio Social I. }\end{array}$ \\
\hline
\end{tabular}

\begin{tabular}{|c|c|c|c|c|c|c|}
\hline \multirow{2}{*}{ PROPÓSITO } & \multirow{2}{*}{ ESTRATEGIA } & \multirow{2}{*}{ ACTIVIDAD } & \multicolumn{2}{|c|}{ RECURSOS } & \multirow{2}{*}{$\begin{array}{l}\text { LUGAR DE } \\
\text { EJECUCIÓN }\end{array}$} & \multirow{2}{*}{ RESPONSABLES } \\
\hline & & & Materiales & Humanos & & \\
\hline & & $\begin{array}{l}\text { Encuentro con los miembros de la } \\
\text { Casa de los Abuelos Angélica } \\
\text { Alvarado a traves de mesas } \\
\text { socializadoras para conocer las } \\
\text { causas de los problemas detectados } \\
\text { en el diagnóstico participativo así } \\
\text { como las consecuencias de dichos } \\
\text { problemas. Se utilizó la técnica del } \\
\text { Arbol del problema. }\end{array}$ & $\begin{array}{l}\text { Cuaderno de Notas. } \\
\text { Lápiz. } \\
\text { Papel bond. } \\
\text { Cámara Fotográfica. }\end{array}$ & $\begin{array}{l}\text { Investigador, miembros de la } \\
\text { casa de los abuelos Angélica } \\
\text { Alvarado y miembros del } \\
\text { Consejo Comunal Territorio } \\
\text { social I. }\end{array}$ & $\begin{array}{l}\text { Casa de los Abuelos } \\
\text { Angélica Alvarado, } \\
\text { calle Colon, sector } \\
\text { Pantano Abajo. }\end{array}$ & $\begin{array}{l}\text { Docente de la UNEFM y } \\
\text { miembros del Consejo } \\
\text { comunal territorio social I. }\end{array}$ \\
\hline $\begin{array}{l}\text { Aplicar } \\
\text { estrategias } \\
\text { de salud en } \\
\text { las áreas de } \\
\text { Microbiología } \\
\text { ' Cardiovascul } \\
\text { ar, Endocrino } \\
\text { y } \\
\text { Enfermedade } \\
\text { s metabólicas } \\
\text { en la casa de } \\
\text { los abuelos } \\
\text { "Angélica } \\
\text { Alvarado". }\end{array}$ & $\begin{array}{l}\text {-Gestionar con el } \\
\text { Ambulatorio Urbano tipo II } \\
\text { Edgar Peña y Secretaria de } \\
\text { Salud la atención de } \\
\text { patologías crónicas y y } \\
\text { detección de micosis } \\
\text { superficiales en los adultos } \\
\text { mayores de la casa de los } \\
\text { Abuelos Angélica Alvarado } \\
\text { del sector Pantano Abajo. } \\
\text {-- Organizar e implementar } \\
\text { las actividades de salud; } \\
\text { Cardiovascular, Endocrino, } \\
\text { detección de micosis y } \\
\text { superficiales Enfermedades Metabólicas } \\
\text { Enfermedas } \\
\text { en la casa de los abuelos } \\
\text { Angélica Alvarado. }\end{array}$ & $\begin{array}{l}\text { Entrega de comunicaciones a los } \\
\text { jefes de División Regional del } \\
\text { Programa Endocrino, enfermedad } \\
\text { metabólica y y cardiovascular } \\
\text { solicitando apoyo para la } \\
\text { implementación de estrategias de } \\
\text { salud en la casa de los abuelos } \\
\text { Angélica Alvarado. } \\
\text {.Entrega de comunicación a la } \\
\text { Coordinación del Ambulatorio } \\
\text { Urbano tipo Il Edgar Peña solicitando } \\
\text { apoyo para la evaluación y control } \\
\text { de patologías crónicas y detección } \\
\text { micosis superficiales en los adultos } \\
\text { mayores de la casa de los abuelos } \\
\text { Angélica Alvarado. } \\
\text {-Reunión con los jefes de división de } \\
\text { la Secretaria de Salud y la } \\
\text { Coordinadora para implementación } \\
\text { de estrategias de salud integral en la } \\
\text { casa de los abuelos. } \\
\text {-Planificación de las actividades de } \\
\text { salud en conjunto con la Licda } \\
\text { Amarilis Ruiz, gerontóloga de la casa } \\
\text { de los abuelos Angélica Alvarado, la } \\
\text { directiva y los miembros del consejo }\end{array}$ & $\begin{array}{l}\text { Cuaderno de Notas. } \\
\text { Lápiz. } \\
\text { Cámara Fotográfica. }\end{array}$ & $\begin{array}{l}\text { Investigadores y personal del } \\
\text { ambulatorio y Jefes de } \\
\text { División de Secretaria de } \\
\text { Salud. }\end{array}$ & $\begin{array}{l}\text { Sector Pantano Abajo } \\
\text { y Hospital } \\
\text { Universitario "Dr. } \\
\text { Alfredo Van Grieken". }\end{array}$ & $\begin{array}{l}\text { Docentes de la UNEFM, } \\
\text { personal del ambulatorio, } \\
\text { secretaria de salud y } \\
\text { consejo comunal. }\end{array}$ \\
\hline
\end{tabular}


SALUD Y VIDA. Revista Arbitrada Interdisciplinaria de Ciencias de la Salud

. Año I. Volumen 1. Enero - Junio 2017. Santa Ana de Coro, Venezuela.

Hecho el depósito de Ley: FA2016000010. ISSN: 2610-8038

FUNDACIÓN KOINONIA (F.K). Santa Ana de Coro, Venezuela.

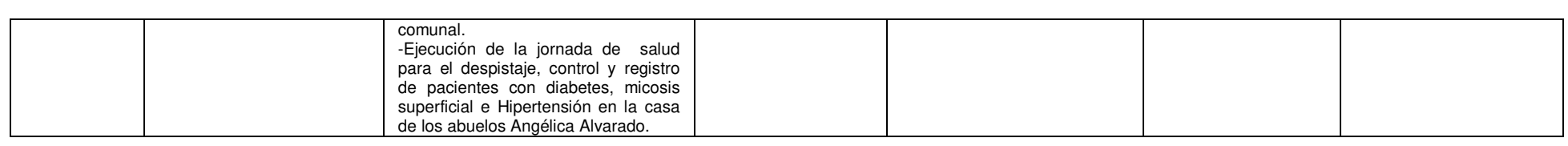

\begin{tabular}{|c|c|c|c|c|c|c|}
\hline \multirow[b]{2}{*}{ PROPÓSITO } & \multirow{2}{*}{ ESTRATEGIA } & \multirow{2}{*}{ ACTIVIDAD } & \multicolumn{2}{|c|}{ RECURSOS } & \multirow{2}{*}{$\begin{array}{l}\text { LUGAR DE } \\
\text { EJECUCIÓN }\end{array}$} & \multirow{2}{*}{ RESPONSABLES } \\
\hline & & & MATERIALES & HUMANOS & & \\
\hline $\begin{array}{l}\text { Efectuar } \\
\text { talleres de } \\
\text { formación en } \\
\text { Proyectos } \\
\text { Socio- } \\
\text { Productivos } \\
\text { para los } \\
\text { adultos de la } \\
\text { mayores de la } \\
\text { casa de los } \\
\text { Abuelos } \\
\text { "Angélica } \\
\text { Alvarado". }\end{array}$ & $\begin{array}{l}\text {-Entregar comunicación a la } \\
\text { Gerencia de Proyectos socio- } \\
\text { productivos de CORFALTUR } \\
\text { para la implementación de } \\
\text { talleres socio-productivos. } \\
\text {-Organizar en conjunto con } \\
\text { CORFALTUR y consejo } \\
\text { comunal los talleres de } \\
\text { formación en proyectos socio- } \\
\text { productivos para los adultos } \\
\text { mayores de la casa de los } \\
\text { abuelos Angélica Alvarado y la } \\
\text { comunidad del sector Pantano } \\
\text { Abajo. }\end{array}$ & $\begin{array}{l}\text {-Visita a Corfaltur para solicitar apoyo } \\
\text { para la realización de los talleres: El } \\
\text { turismo como factor productivo y el } \\
\text { Manual de Proyectos Socio-productivos } \\
\text { dirigido a los adultos mayores de la casa } \\
\text { de los abuelos Angélica Alvarado y la } \\
\text { comunidad en general del sector Pantano } \\
\text { Abajo. } \\
\text {-Reunión con la socióloga Vanessa } \\
\text { Sterling coordinadora de CORFALTUR } \\
\text { para la organización de los talleres: El } \\
\text { turismo como factor productivo y el } \\
\text { manual sobre proyectos socio- } \\
\text { productivos para los adultos mayores de } \\
\text { la casa de los abuelos Angélica Alvarado } \\
\text { y la comunidad en general del sector } \\
\text { Pantano. } \\
\text {-Ejecución de los talleres; El turismo } \\
\text { como factor productivo y el manual para } \\
\text { proyectos socio-productivos en la casa de } \\
\text { los abuelos Angélica Alvarado y la } \\
\text { comunidad del sector Pantano Abajo. }\end{array}$ & $\begin{array}{l}\text { Cuaderno de Notas. } \\
\text { Lápiz. } \\
\text { Cámara Fotográfica. }\end{array}$ & $\begin{array}{l}\text { Investigadores, } \\
\text { del consejo combros } \\
\text { territorio social I y el } \\
\text { personal de COLFALTUR. }\end{array}$ & $\begin{array}{lr}\text { Calle } & \text { Zamora, } \\
\text { sector } & \text { Casco } \\
\text { Histórico de Santa } \\
\text { Ana de Coro. }\end{array}$ & $\begin{array}{l}\text { Docentes de la UNEFM y } \\
\text { miembros del consejo } \\
\text { comunal territorio social I }\end{array}$ \\
\hline $\begin{array}{l}\text { - Realizar } \\
\text { actividades } \\
\text { físicas y de } \\
\text { recreación los } \\
\text { para } \\
\text { adultos los la } \\
\text { mayores de la } \\
\text { casa de los } \\
\text { abuelos } \\
\text { "Angélica } \\
\text { Alvarado" para } \\
\text { fomentar } \\
\text { hábitos de } \\
\text { vida saludable. }\end{array}$ & $\begin{array}{l}\text {-Entregar comunicación dirigida } \\
\text { al Coordinador de Deportes de } \\
\text { Municipalización UNEFM, } \\
\text { Lcdo. Rubén Molina en } \\
\text { solicitud de apoyo para la } \\
\text { realización de actividades } \\
\text { físicas y recreativas en la casa } \\
\text { de los Abuelos Angélica } \\
\text { Alvarado. } \\
\text {-Planificar y organizar con el } \\
\text { coordinador de Deportes de } \\
\text { Municipalización de la UNEFM, } \\
\text { la gerontóloga y Consejo } \\
\text { Comunal las actividades } \\
\text { físicas y recreativas en la casa } \\
\text { de los Abuelos Angélica } \\
\text { Alvarado Ado }\end{array}$ & $\begin{array}{l}\text {-Reunión con el Lcdo. Rubén Molina } \\
\text { Coordinador de Deportes de } \\
\text { Municipalización de la UNEFM para la } \\
\text { planificación de jornadas deportivas en la } \\
\text { casa de los abuelos Angélica Alvarado. } \\
\text {-Reunión con la Lcda. Amarilis Ruiz } \\
\text { gerontóloga de la casa de los Abuelos } \\
\text { Angélica Alvarado y el Consejo Comunal } \\
\text { Territorio Social I, para la organización de } \\
\text { las jornadas de actividad física y de } \\
\text { recreación. } \\
\text {-Ejecución de la jornada de salud } \\
\text { cardiovascular a través de actividades } \\
\text { físicas y recreativas en la casa de los } \\
\text { abuelos Angélica Alvarado. } \\
\text { - Caminata ecológica y actividades } \\
\text { recreativas con los adultos mayores de la } \\
\text { casa de los abuelos y los grupos sociales } \\
\text { que hacen vida en el ambulatorio "Edgar } \\
\text { Peña." }\end{array}$ & $\begin{array}{l}\text { Cámara Fotográfica } \\
\text { Materiales deportivos } \\
\text { Tensiómetros } \\
\text { Medicamentos. } \\
\text { Refrigerio. } \\
\text { Cuaderno de Registro. } \\
\text { Trípticos. }\end{array}$ & $\begin{array}{l}\text { Investigadores, } \\
\text { coordinador de Deportes y } \\
\text { docentes de deportes de la } \\
\text { UNEFM. }\end{array}$ & $\begin{array}{lrr}\text { Casa } & \text { de } & \text { los } \\
\text { Abuelos } & \text { Angélica } \\
\text { Alvarado, ralle } & \text { calle } \\
\text { Colón, } & \text { sector } \\
\text { Pantano Abajo }\end{array}$ & $\begin{array}{l}\text { Docentes de la UNEFM, } \\
\text { coordinador de Deportes } \\
\text { y docentes de deportes } \\
\text { de la UNEFM, Licenciada } \\
\text { Amarilis Ruiz y Consejo } \\
\text { Comunal. }\end{array}$ \\
\hline
\end{tabular}



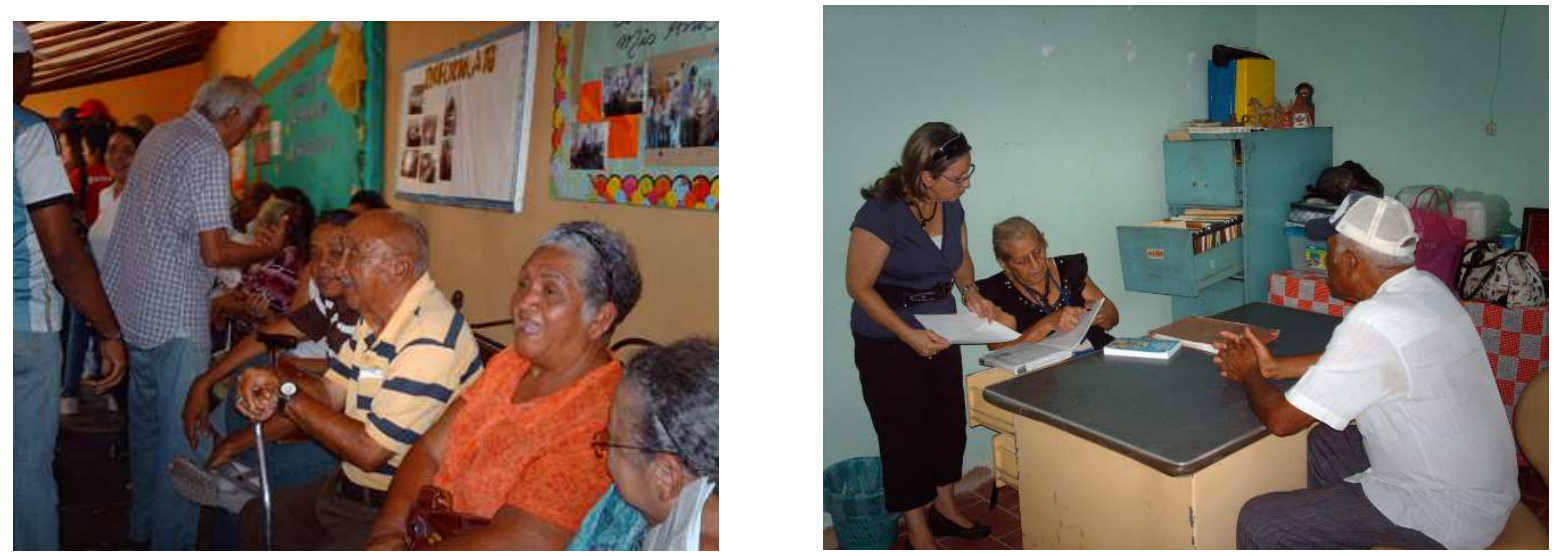

Mesas socializadoras con los adultos mayores para la determinación de problemáticas
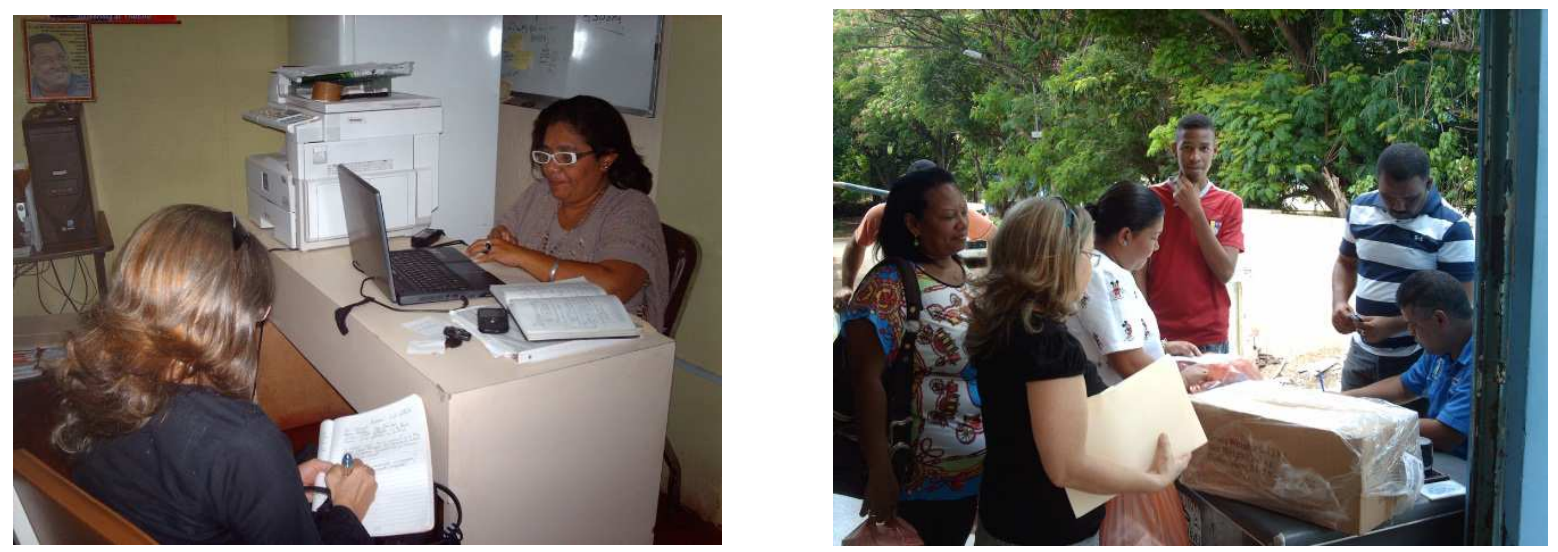

Reunión en Secretaria de Salud y donación de medicamentos para los Adultos mayores de la casa "Angélica Alvarado" 


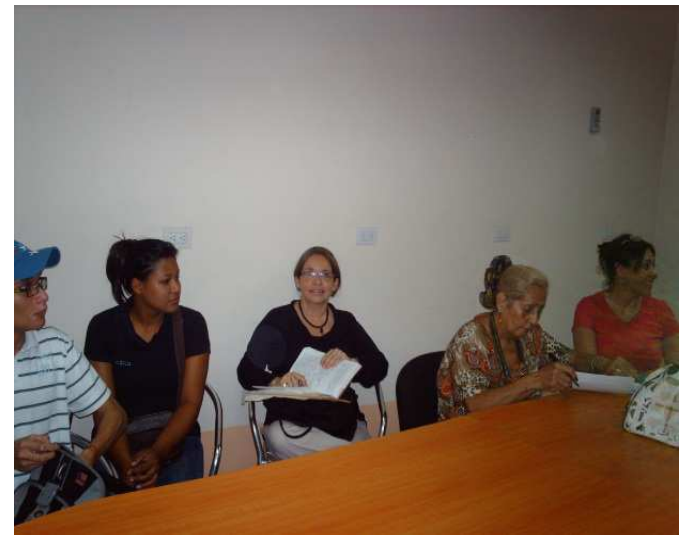

Taller en CORFALTUR

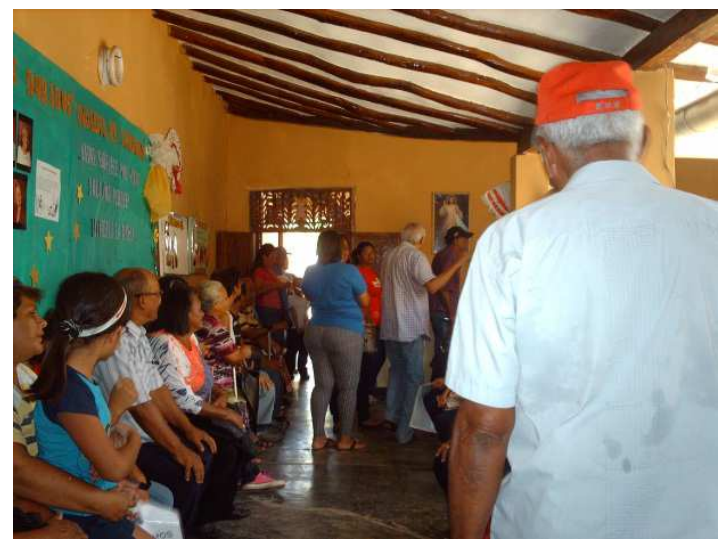

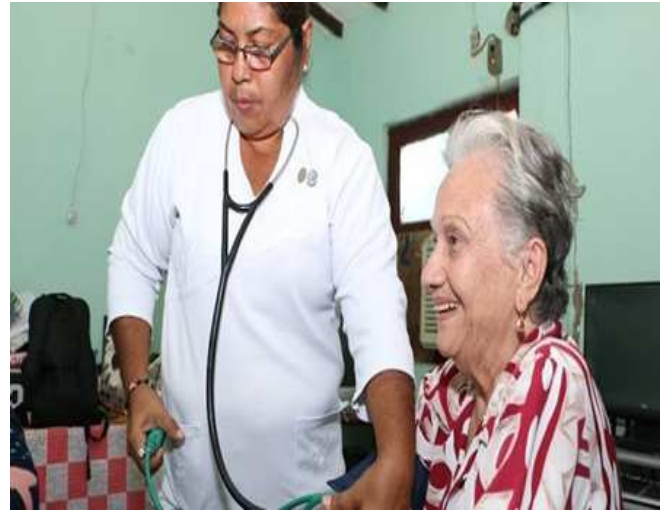

Jornadas de salud

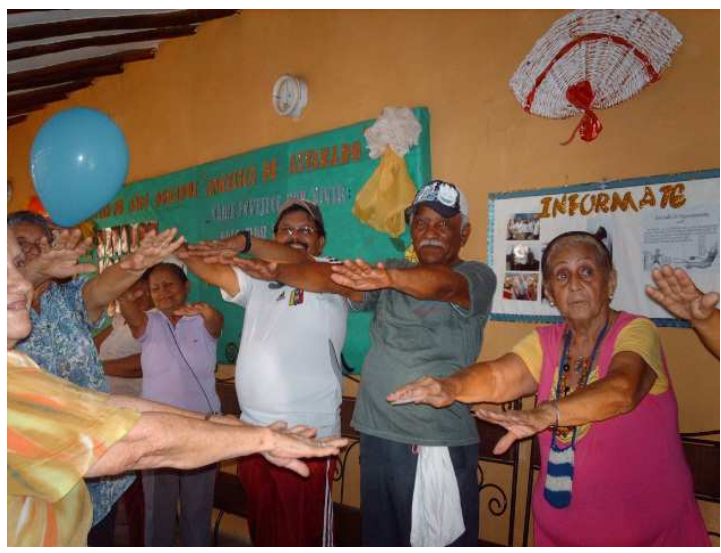

Actividades Deportivas y Recreativas con los Adultos mayores de la Casa "Angélica Alvarado" 

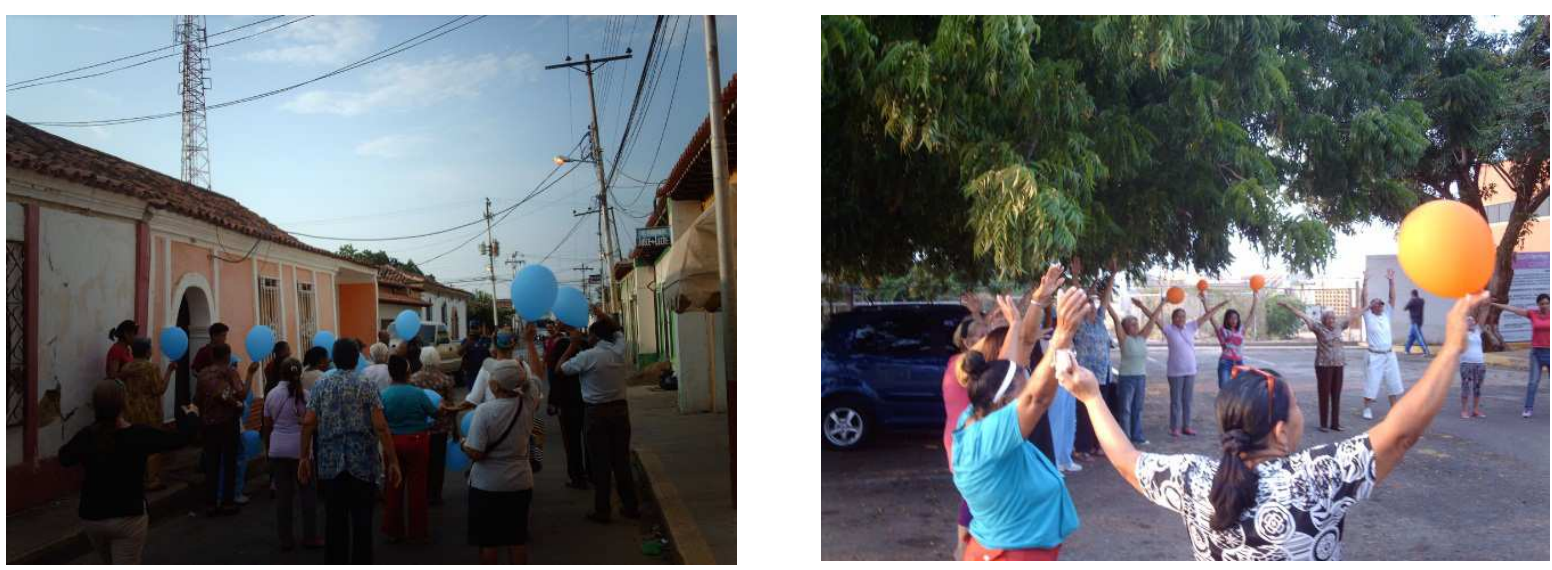

Caminata Ecológica y Actividades Recreativas con los adultos mayores De la casa de los abuelos "Angélica Alvarado"

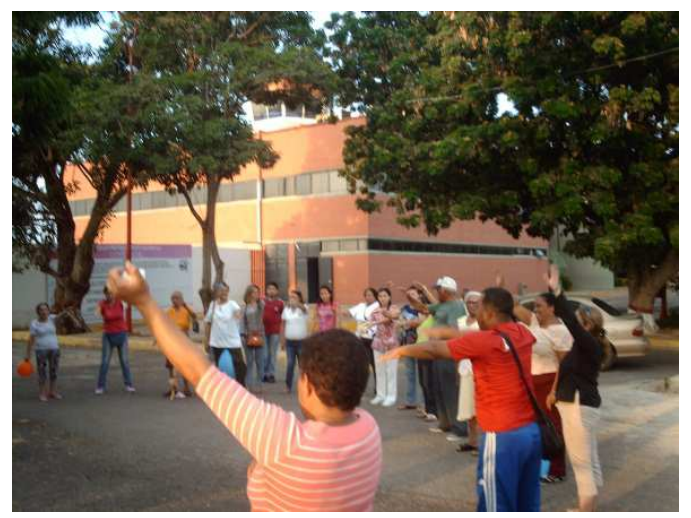

Actividades Deportivas con los adultos mayores De la casa "Angélica Alvarado" 


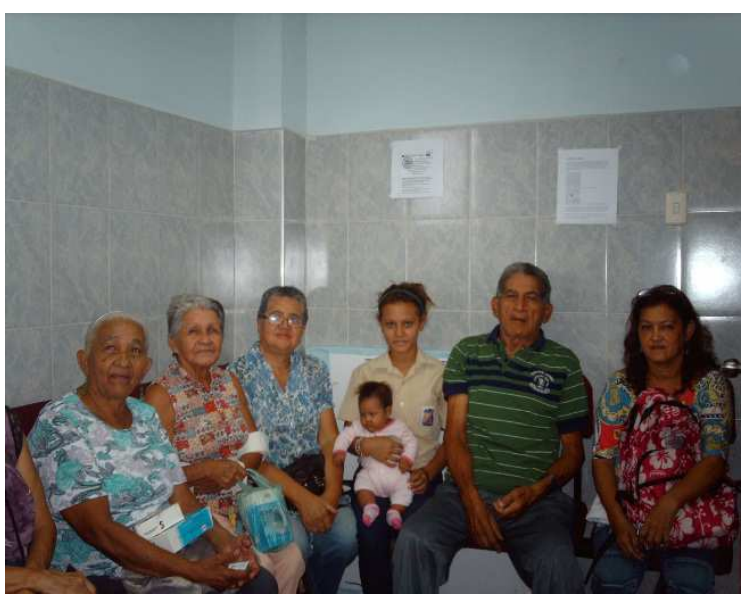

Entrega de Glucómetros y tratamiento hipertensivo a los adultos Mayores de la casa "Angélica Alvarado"
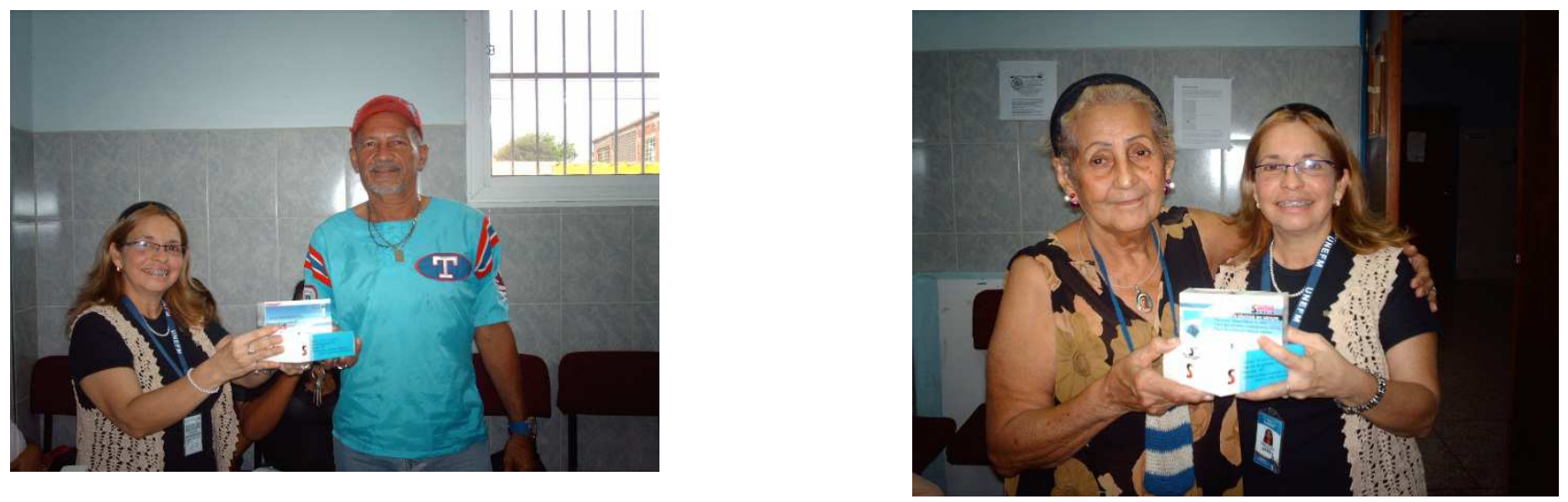

Entrega de los Glucómetros a los adultos mayores en el ambulatorio "Edgar Peña" 


\section{Reflexiones Finales}

-Con la intervención comunitaria efectuada en la Casa de los Abuelos "Angélica Alvarado" se pudo detectar la falta de atención integral; a la salud, la actividad física-recreación y la formación en proyectos socio-productivos los cuales afectaban la calidad de vida de los adultos mayores. Lo que conllevo a planificar estrategias y actividades de Atención Primaria de Salud a través de la Secretaria de Salud y el Ambulatorio Urbano Tipo II "Edgar Peña", los Consejos Comunales y la comunidad en general en la casa de los Abuelos "Angélica Alvarado", lo que permitió que desde el punto de vista social la intervención beneficiara a la comunidad creando consciencia acerca de las problemáticas de salud pública que representan la diabetes Mellitus, las micosis superficiales y la hipertensión tanto a nivel mundial como a nivel local debido a sus altos índices de morbi-mortalidad como de sus complicaciones.

-La intervención comunitaria permitió resaltar la importancia de la realización de actividades físicas para la prevención y promoción de la salud a través de hábitos saludables con la realización de actividades físicas y recreativas que permitan eliminar el sedentarismo lo cual favorece la preservación y el mantenimiento de la salud del adulto mayor.

-Se atendió a los adultos mayores en su proceso de formación para la participación activa dentro de la comunidad a través de la implementación de talleres en proyectos socio-productivos que no solo beneficiaran desde el punto de vista económico a los adultos mayores sino que permitirán en un futuro contribuir al desarrollo económico y social de la comunidad del sector Pantano Abajo con la creación de empresas comunitarias. El propósito trazado de la investigación se cumplió mediante el estudio, reconocimiento de las problemáticas más sentidas por parte de los adultos mayores de la casa de los Abuelos "Angélica Alvarado" en relación a la atención integral de la salud y se logró concientizar además de motivar la participación y colaboración de diferentes instituciones del Estado, la Comuna Heroína "Josefa Camejo", el Consejo Comunal Territorio social I y la comunidad en general para la mejora de la calidad de vida del adulto mayor. 


\section{Referencias Consultadas}

1. Bautista, M. (2004). Manual de Metodología de la Investigación. Edit. Talleres Litográficos. Caracas. Venezuela.

2. Beauregard, S. (1987). Arch. Dermatol. J. Gerontol.

3. Blanco, H. (2010). El Pantano de Coro. Editorial Faro de Falcón.

4. Brownlee, M. (1991). Glycosylation Products as Toxic mediators of Diabetic Complications. Annu. Rev. Med.

5. Chacón, M. (2009). El Desarrollo Comunitario. Revista Digital de innovación y Experiencias Educativas. Número 29. Depósito Legal: GR2922/20017

6. Comuna Socialista Heroína Josefa Camejo (2013).Carta Fundacional. Registro del Poder Popular.

7. Duno, R. (2011). Casa del Abuelo Angélica Alvarado. Disponible en: Casadelabueloangelicaalvarado.blogspot.com.

8. Gollete, G. (1988). La Investigación Acción; Funciones, fundamentos e instrumentación. Edit. Laertes. Barcelona. España.

9. Hall, W. (2013). Envejecimiento Saludable. U. S. National Institutes of Health.

10. Instituto del Patrimonio Cultural (2005). Catálogo del Patrimonio Cultural Venezolano, Región Centro -Occidental. Editorial Intenso Offsot. Caracas.

11. Instituto Nacional de Estadística del Estado Falcón (INEF) 2015.

12. Koelting, K. (1984). Paradigmas de Investigación Educativa. Edit. Panapo.

13. Kuetlner, K. (1992). Articular Cartilage and Osteoarthritis. Editor. Processes of Aging. N.Y.: Atherton Press.

14. Kurt,L. (1951). La Teoría de Campo en la Ciencia Social. Edit. Paidos. Buenos Aires. Argentina.

15. Organización de las Naciones Unidas para la Educación, la Ciencia y la Cultura (UNESCO1993).

16. Organización Mundial de la Salud (2002). Active Ageing a Policy Framework. Madrid. España.

17. Ribera, J. (2009). Geriatría en Atención Primaria. Editorial Grupo Aula Médica. Madrid. España.

18. Rose, M. (1991). Evolutionary Biology of Ageing. Editor. Processes of Aging. N.Y.: Atherton Press.

19. Swinne, C. (1992). Am J Cardiol. J. Gerontol.

20.Tockman, M. (1998). Am Rev Respir Dis. (Suppl). J. Gerontol.

21. Yaar, M. (2001). Envejecimiento de la Piel. Editores: Dermatología en Medicina General. 5ta edición. Buenos Aires.

22. Yates, L. (2008). Exceptional Longevity in Men: Modifiable Factors Associated with Survival and Function to Age 90 years. Edit. Archives of Internal Medicine. 